this, PSV trials were well tolerated in the majority with active weaning in pressure support subsequently achieved. Individual screening criteria were not associated with PSV failure. Of clinical significance, failure to sustain a PSV trial could be an early indicator of prolonged mechanical ventilation and ICU mortality, and predictive characteristics of this warrant further investigation. Application of screening criteria, as reported in weaning literature, may delay initiation of weaning in some patients.

\section{S137 FAILURE OF NICE GUIDANCE CG83 IMPLEMENTATION: NATIONAL UK SURVEY OF REHABILITATION SERVICES FOR SURVIVORS OF CRITICAL ILLNESS}

${ }^{1} \mathrm{~B}$ Connolly, ${ }^{2} \mathrm{~L}$ Denehy, ${ }^{1} \mathrm{~J}$ Moxham, ${ }^{3} \mathrm{~N}$ Hart; ${ }^{1}$ Department of Asthma, Allergy \& Respiratory Science, Division of Asthma, Allergy and Lung Biology, King's College London, London, UK; ' ${ }^{2}$ Department of Physiotherapy, School of Health Sciences, University of Melbourne, Melbourne, Australia; ${ }^{3}$ Lane Fox Clinical Respiratory Physiology Research Unit, St. Thomas' Hospital, Guy's \& St. Thomas' NHS Foundation Trust, London, UK

\subsection{6/thoraxjnl-2013-204457.144}

Introduction National guidelines advocating multidisciplinary rehabilitation delivered throughout the continuum of recovery following critical illness were published in 2009 (NICE CG83). However, lack of supporting evidence for these recommendations has resulted in inconsistent implementation, particularly in the post hospital discharge phase. As expected, nursing and medical staff have been surveyed for involvement in intensive care unit (ICU) follow-up with previous surveys of physiotherapy practice focussed on within-ICU rehabilitation practice. This survey aimed to characterise post critical illness follow-up and rehabilitation following hospital discharge.

Method A predominantly closed-question, physiotherapy-specific postal survey distributed to senior critical care clinicians at UK hospitals with a listed ICU, excluding specialist-only units, and coded to facilitate respondent identification.

Abstract S137 Table 1. Barriers to availability of post hospital discharge rehabilitation programmes for post critical illness patients.

\begin{tabular}{lll}
\hline Barrier & $\begin{array}{l}\text { Frequency } \\
\text { reported } \\
\text { overall } \mathbf{n}(\%)\end{array}$ & $\begin{array}{l}\text { Frequency reported } \\
\text { as main barrier }\end{array}$ \\
\hline Lack of funding & $147(90.7)$ & $98(63.6)$ \\
Lack of sufficient staff & $127(78.4)$ & $17(11.0)$ \\
Resources prioritised to other & $70(43.2)$ & $4(2.6)$ \\
patient groups/clinical areas & & \\
Not considered required service & $66(40.7)$ & $22(14.3)$ \\
at managerial level & & \\
Lack of available space & $50(30.9)$ & $2(1.3)$ \\
Insufficient patient numbers to justify & $34(21.0)$ & $10(6.5)$ \\
Extra-contractual (out-of-area) patient caseload & $15(9.3)$ & $0(0.0)$ \\
Lack of trained staff & $12(7.4)$ & $0(0.0)$ \\
No evidence & $4(2.5)$ & $0(0.0)$ \\
Not sure what to include in a programme & $2(1.2)$ & $0(0.0)$ \\
Other (time constraints) & $1(0.6)$ & $1(0.6)$ \\
\hline For frequency of reported barriers overall, $\mathrm{n}=162$ responses. & For frequency of reporting \\
as main barrier, $\mathrm{n}=154$ responses. & & \\
&
\end{tabular}

Results Physiotherapists at 240 identified ICUs were sent surveys. 182 surveys were returned $(75.8 \%$ response rate), including one blank survey. $36.5 \%$ were from university teaching hospitals, $63.5 \%$ from district general hospitals. Forty-eight centres reported follow-up services at 2-3months, the majority as clinics $(39 / 48,81.3 \%)$. Physiotherapists were involved in 43 follow-up services, albeit in a third of cases, on a referral-only basis. Critical care nursing staff were the main other clinician involved. Health-related quality of life $(83.3 \%)$ and psychological status $(81.3 \%)$ were the main items addressed, with exercise capacity reviewed in almost $60 \%$ of cases. Only $12 / 182(6.6 \%)$ centres reported post hospital discharge rehabilitation programmes, all including an exercise component, but only four offering education topics. Substantial variation existed between programmes regarding eligibility, delivery format, structure, content and evaluation. Where rehabilitation programmes were not available, barriers to offering this service were detailed (Table 1). Lack of funding was the most frequently reported, and the main barrier listed. The majority of respondents (96/169; 56.8\%) reported referral of post critical illness patients into alternative rehabilitation streams, predominantly accessing community and domiciliary in-patient or out-patient services.

Conclusion Data from this survey demonstrated a low level of available follow-up and rehabilitation services for post critical illness patients following hospital discharge. This reflects a lack of implementation of high-profile national guidelines, the main reason for which was reported to be lack of funding. Further investment in these services is required.

\section{Mechanisms in pulmonary vascular disease}

\section{S138 BLOOD OUTGROWTH ENDOTHELIAL CELLS ISOLATED FROM PATIENTS WITH PULMONARY ARTERIAL HYPERTENSION POSSESS LESS CAVEOLAE AND REDUCED CAVIN-2 EXPRESSION}

${ }^{1} \mathrm{BJ}$ Dunmore, ${ }^{2} \mathrm{G}$ Howard, ${ }^{1} \mathrm{~A}$ Crosby, ${ }^{2} \mathrm{BJ}$ Nichols, ${ }^{1} \mathrm{NW}$ Morrell; ${ }^{1}$ University of Cambridge, Cambridge, United Kingdom; ${ }^{2}$ MRC Laboratory of Molecular Biology, Cambridge, United Kingdom

\subsection{6/thoraxjnl-2013-204457.145}

Pulmonary arterial hypertension $(\mathrm{PAH})$ is a progressive disease characterised by a marked elevation in pulmonary arterial pressure due to increased muscularisation and obliteration of small pulmonary arteries. The majority of heritable PAH cases are due to mutations in members of the transforming growth factor- $\beta$ (TGF $\beta$ ) receptor signalling pathway. Although predominantly associated with mutations in the bone morphogenetic protein type II receptor (BMPR-II) genetic variations causing disease have recently been identified in caveolin-1, the major component of caveolae generation. Caveolae are flask shaped invaginations in the plasma membrane that play an important role in many endothelial cell functions including vesicular trafficking and signalling. Correct caveolae formation in the lung has recently been shown to require serum deprivation protein response (SDPR), also known as cavin-2. Using blood outgrowth endothelial cells (BOECs) isolated from patients with $\mathrm{PAH}$ we sought to determine the expression of cavin-2 and caveolae formation.

Methods Blood outgrowth endothelial cells (BOECs) were isolated from control subjects and individuals with $\mathrm{PAH}$, including BMPR-II mutation and idiopathic patients. Following transmission electron microscopy (TEM) the number of caveolae and depth of invaginations were assessed in ten cells from each cell line. Protein expression of caveolin-1, cavin-1 and cavin-2 were assessed by western blotting. Caveolin-1 and cavin-2 knockout 